\title{
Reflection of a Convener, PANCON-7
}

\author{
Shakya DR
}

Professor, Department of Psychiatry, BPKIHS, Dharan, Nepal

E-mail *Corresponding author: drdhanashakya@yahoo.com

Dear Editor,

I am writing here as Convener, Scientific Program and Publication sub-committee of the Seventh National Conference of Psychiatrists' Association of Nepal 2019 (PANCON-7) held in March 7-8, 2019 in B. P. Koirala Institute of Health Sciences (BPKIHS), Dharan, Nepal. This is to reflect briefly to the Scientific Program and Publication in the PANCON-7.

When the Executive committee of the Psychiatrists' Association of Nepal (PAN) approached the Department of Psychiatry, BPKIHS for hosting the PANCON-7, there was a great challenge for making it academically rich, professionally refreshing and strategically influential one. Our organizing committee chose the theme 'Many faces of Psychiatry in Changing World' parallel to that of the World Mental Health Day 2018, i.e. 'Young people and Mental health in Changing World'. We put every effort to call and invite for related presentations, including: key-note speech, guest lectures, symposium, workshops, free oral papers and posters making overall of 60 psychiatric and mental health issues.

We were privileged to have: Prof. Dr. Mohan Isaac (The University of Western Australia, Perth) for thematic speech, Prof. Dr. S. K. Khandelwal (AIIMS, New Delhi, India) and Prof. Dr. Shekhar Seshadri (NIMHANS, Bengaluru, India) for theme related guest lectures and Prof. Dr. Indira Sharma (Heritage Institute of Medical Sciences, Varanasi, India) for a related guest lecture. The insight thrown by Prof. Mohan Isaac into the current context of changing world as globalization and its relationship to mental health is a real gem of the meet. Current trend characterized by rapid technological advances, easier, cheaper and faster communication, digital revolution and acceleration in many respects has brought us both opportunities and challenges in all aspects including mental health. Prof. Isaac pointed out the 'Recovery' oriented mental health services, early intervention in psychosis, continuing universal stigma, anti-psychiatry movement, digital/tele-psychiatry and declining suicide rates as some remarkable features of the current world in the field of mental health and psychiatry. The close look by Prof. S. K. Khandelwal into the context of developing world, including Nepal, is other remarkable insight. Prof. Khandelwal highlighted that the great challenge was lack of preparation in developing nations and people there to manage the precipitated advances, changes and technology which result into adverse consequences, including mental health adversity. More intensive perspective raised for growing child and adolescent population by Prof. Shekhar Seshadri and women's mental health from point of view of sexual harassment/misconduct at workplace by Prof. Indira Sharma were other significant presentations of the meet.

Our senior psychiatrist Dr. Kapil Dev Upadhyaya's deliberation on 'Spirituality and Mental health' added other great facet. A symposium about our (psychiatrists' economic) survival in Nepal is much needed one and I trust that the recommendations developed out of the then interactions will be submitted to related authorities including new executive committee of the PAN for further steps. Similarly, sessions on substance, music, dual diagnosis, psychiatry career and Government programs on mental health were other remarkable ones. Much awaited presentations of Prof. Dr. Mahendra Kumar Nepal (Australia) regarding 'Adjustment disorder' and of Dr. 
Ranjan Thapa on 'Social media' could not take place at last moment due to unavoidable circumstances. Altogether, we had 21 posters, 17 oral, 10 lectures, 4 symposiums and 1 half day workshop; making 60 individual presentations. There is a remarkable representation of relatively young but promising and intellectually high brains as speakers in this conference. We had a special half day workshop on 'Psychosexual disorders' by a team of Prof. Dr. T. S. S. Rao (JSSMC, JSS University, Mysore, Karnataka), Dr. Ajayan Verughese (Kerala, India) and Dr. Vineet Malhotra (New Delhi, India).

We hope with the help of our wise chairpersons like: Drs. D. R. B. Kunwar, V. D. Sharma, Kapil Dev Upadhyaya, S. K. Khandelwal, Nabaral Koirala, Saroj Ojha, S. N. Pradhan, Ajit Gurung, Rabi Shakya, Shailendra Raj Adhikari, Baikuntha Raj Adhikari, Namrata Mahara, Nirmal Lamichhane, Sami Lama, Pradeep Man Singh, Sandip Subedi, Rajan Sharma, Roshan Pokharel, Manoj Dhungana in all our sessions; we met, got to know each other, shared our ideas, experiences and knowledge, we discussed, came up with some way out to face challenges of this changing current time and prove it academically fruitful meet.

Felicitation and 'life-time achievement award' to our respected senior most alive psychiatrist Dr. D. R. B. Kunwar sir was a glorious part of this event. A 4-membered evaluation team declared Dr. Pawan Sharma's presentation regarding 'Suicide registry' as the best of the conference. PAN also awarded Dr. Ajay Risal for his published journal article.

We also put our earnest effort to make the Souvenir remarkable by including our PAN member Directory. Despite of our sincere efforts, it could not include many and as Convener, I humbly request the upcoming PAN Executive to collect the rest and maintain its data. I appreciate all authorities for good wishes and messages and all authors for the articles.

Hosting a national event like this in Dharan was like our effort to cover many people with a small blanket. We felt when one corner is covered, other side left out. Whatever be the reason, we look forward to creative feedback/ suggestions. We believe with active participation, discussion and involvement of all the delegates and speakers in those two days made the event academically a true conference; off course, not forgetting to make note of beauty of this city and institute and boost our personal and professional ties.

From the Organizing committee, thanks to: the PAN for entrusting us for the national conference, all respected speakers for generous help, all delegates for active participation, BPKIHS authorities for needful cooperation and all seen unseen hands, hearts and brains contributing to make it a success. We also extend our congratulations to outgoing PAN Executive team of Dr. Arun Raj Kunwar and good luck to incoming team of Prof. Dr. S. N. Pradhan for successful tenure.

At last, wish our respected DRB Kunwar Sir, all our senior and guest speakers and PAN members a healthy, wealthy and prosperous life ahead. Thanks.

Best Regards, DRS. 